\title{
Fexofenadine HCl Immediate Release Tablets: In vitro Characterization and Evaluation of Excipients
}

\author{
Shahriar Ahmed ${ }^{1}$, Mehrina Nazmi ${ }^{1}$, Ikramul Hasan ${ }^{1}$, Sabiha Sultana ${ }^{1}$, Shimul Haldar ${ }^{2}$ \\ and Md. Selim Reza ${ }^{2}$ \\ ${ }^{1}$ Department of Pharmacy, University of Asia Pacific, Dhanmondi, Dhaka-1209, Bangladesh \\ ${ }^{2}$ Department of Pharmaceutical Technology, Faculty of Pharmacy, University of Dhaka, Dhaka-1000, Bangladesh.
}

\begin{abstract}
Fexofenadine $\mathrm{HCl}$ immediate release tablets were designed to increase the dissolution rate by using superdisintegrants. Different formulations of Fexofenadine $\mathrm{HCl}$ were prepared by direct compression method. These formulations were evaluated for hardness, thickness, friability, weight variation, disintegration time, and in vitro dissolution study. The drug release from the formulations were studied according to USP specification (USP paddle method at $50 \mathrm{rpm}$ for 60 minutes) maintaining the temperature to $37^{\circ} \mathrm{C}$. Sodium starch glycolate, cross carmellose sodium, crospovidone (kollidon CL), ludiflash and xanthan gum were used in 3\%, 6\% and 8\% concentrations as superdisintegrants. Thus, the ratio of superdisintegrants was changed whereas all the other excipients as well as the active drug (Fexofenadine $\mathrm{HCl}$ ) remained same in every formulation. Here, $0.001 \mathrm{~N} \mathrm{HCl}$ was used as dissolution medium according to USP and absorbances were determined by using UV spectrophotometer at $217 \mathrm{~nm}$. The F-3 and F-6 formulation prepared by $8 \%$ of Sodium starch glycolate and 8\% of Cross carmellose sodium showed $99.99 \%$ drug release within 30 minutes and 45 minutes, respectively. The disintegration times of F-3 and F-6 formulation were within 9 seconds. The interactions between drug and excipients were characterized by FTIR spectroscopic study.
\end{abstract}

Key words: Fexofenadine $\mathrm{HCl}$, Sodium starch glycolate, Cross-carmellose sodium, Kollidon CL, Ludiflash, Xanthan gum, immediate release tablet.

\section{Introduction}

isintegrants are agents added to tablet formulations to promote the breakup of the tablet into smaller fragments in an aqueous environment thereby increasing the available surface area and promoting a more rapid release of the drug substance. They promote moisture penetration and dispersion of the tablet matrix. In recent years, several newer agents have been developed known as "Superdisintegrants". These newer substances are more effective at lower concentrations with greater disintegrating efficiency and mechanical strength (Bhowmik et al., 2010). An important variable in any tablet system is the rate at which the drug substance dissolves and for many solid dosage forms, disintegration precedes drug dissolution. Hence, the proper choice of disintegrants and its consistency of performance are of critical importance to the formulation development of such tablets (Zhao et al., 2005). Superdisintegrants (Shangraw et al., 1980) such as Cross carmellose sodium (CCS),
Sodium starch glycolate (SSG) and Kollidon CL (KCL) are now frequently used in tablet formulations to improve the rate and extent of tablet disintegration thereby increasing the rate of drug dissolution.

Fexofenadine $\mathrm{HCl}$ (FFN) is a second-generation nonsedating histamine $\mathrm{H}_{1}$ receptor antagonist widely used in seasonal allergic rhinitis (Peter et al., 2003). The purpose of the present study was to compare the effect of mode of addition of different superdisintegrants (with various concentrations) and evaluate their effect on dissolution of Fexofenadine $\mathrm{HCl}$ (slightly soluble) in $0.001 \mathrm{~N} \mathrm{HCl}$ as specified in the compendia. Several attempts using different superdisintegrants with various concentrations have been made by direct compression to prepare several formulations of Fexofenadine $\mathrm{HCl}$ immediate release solid dosage forms with improved dissolution properties. Thus, the rationality of the ongoing study lies in meeting the challenge to increase the dissolution profile by decreasing the disintegration time. 


\section{Materials and Methods}

\section{Materials}

Fexofenadine $\mathrm{HCl}$, Avicel Ph 101, Ludiflash (LD) were obtained as gift samples from Incepta Pharmaceutical Ltd., Bangladesh. Sodium starch glycolate (SSG), Cross carmellose sodium (CCS) and Kollidon CL (KCL), Aerosil, Xanthan Gum (XG), Maize Starch, Magnesium stearate were obtained as gift samples from ACI Pharmaceutical Ltd., Bangladesh and other reagents were of analytical grade.

\section{Methods}

Preparation of standard solutions: Stock solutions of Fexofenadine $\mathrm{HCl}$ were prepared by dissolving the drug in $0.001 \mathrm{~N} \mathrm{HCl}$. These stock solutions were diluted to desired strengths by buffer solution $(0.001 \mathrm{~N} \mathrm{HCl})$ to get the working standard solution.

Formulation design: Orally disintegrating tablets were prepared by direct compression method using single punch tablet machine. The formulations were developed by using various ratios.
Formulation design for orally disintegrating tablet by direct compression using superdisintegrants: The superdisintegrants in 3\%, 6\%, and 8\% concentrations were used to develop the tablets. All the ingredients were passed through sieve having mesh no. 40. All the ingredients were cogrounded in a moter pestle. Finally aerosil and magnesium stearate were added and mixed for 5 minutes. The mixed blend of excipients was compressed using a single punch machine to produce convex faced tablets weighing 155, 159 and $163 \mathrm{mg}$ for 3\%, 6\% and 8\% concentrations, respectively with an average of $2.5 \mathrm{~mm}$ thickness. A minimum 35 tablets were prepared for each formulation. The compression force and compression time were 2 tonnes and 15 seconds, respectivly. Before compression, the surfaces of the die and punches were lubricated with Magnesium Stearate. All the preparations were stored in airtight containers at room temperature for further study. Effect of different superdisintegrants with various concentrations was used to study in vitro dissolution characteristics and disintegration time of the formulation (Table 1).

Table 1. Formulations (F1-F15) of different batches by using superdisintegrants-direct compression (quantity in mg).

\begin{tabular}{|c|c|c|c|c|c|c|c|c|c|c|c|c|c|c|c|}
\hline Ingredients & $\mathrm{F} 1$ & F2 & F3 & $\mathrm{F} 4$ & F5 & F6 & F7 & F8 & F9 & F10 & F11 & F12 & F13 & F14 & F15 \\
\hline FFN (drug) & 60 & 60 & 60 & 60 & 60 & 60 & 60 & 60 & 60 & 60 & 60 & 60 & 60 & 60 & 60 \\
\hline SSG & 4.6 & 9.6 & 13.08 & - & - & - & - & - & - & - & - & - & - & - & - \\
\hline CCS & - & - & - & 4.6 & 9.6 & 13.08 & - & - & - & - & - & - & - & - & - \\
\hline KCL & - & - & - & - & - & - & 4.6 & 9.6 & 13.08 & - & - & - & - & - & - \\
\hline LD & - & - & - & - & - & - & - & - & - & 4.6 & 9.6 & 13.08 & - & - & - \\
\hline XG & - & - & - & - & - & - & - & - & - & - & - & - & 4.6 & 9.6 & 13.08 \\
\hline Avicel Ph101 & 80 & 80 & 80 & 80 & 80 & 80 & 80 & 80 & 80 & 80 & 80 & 80 & 80 & 80 & 80 \\
\hline Maize Starch & 4.4 & 4.4 & 4.4 & 4.4 & 4.4 & 4.4 & 4.4 & 4.4 & 4.4 & 4.4 & 4.4 & 4.4 & 4.4 & 4.4 & 4.4 \\
\hline Aerosil & 3 & 3 & 3 & 3 & 3 & 3 & 3 & 3 & 3 & 3 & 3 & 3 & 3 & 3 & 3 \\
\hline Mg-stearate & 3 & 3 & 3 & 3 & 3 & 3 & 3 & 3 & 3 & 3 & 3 & 3 & 3 & 3 & 3 \\
\hline Total & 155 & 160 & 163.5 & 155 & 160 & 163.5 & 155 & 160 & 163.5 & 155 & 160 & 163.5 & 155 & 160 & 163.5 \\
\hline
\end{tabular}

\section{Evaluation of orally disintegrating tablets}

Thickness and diameter: The thickness and diameter of the prepared tablets were measured using slide calipers. These two parameters were expressed in mm.

Weight variation: Weight variation was determined by weighing 20 tablets individually. The average weight and percent variation of tablet was calculated individually.

Hardness and crushing strength: Hardness was determined by taking ten tablets from each formulation, using a Veego tablet hardness tester and the average of applied pressure (kgf) for crushing the tablet was determined.

Friability: The friability of the tablets were determined by friabilator. Initially 20 tablets $\left(\mathrm{W}_{\mathrm{o}}\right)$ were weighed and placed in a friability tester, which was rotated for 4 minutes at $25 \mathrm{rpm}$. After dusting, the total remaining mass of tablets (Wt) was recorded and the percent friability was calculated by

$$
F=100 \times \frac{\mathrm{Wo}-\mathrm{Wt}}{\mathrm{Wo}}
$$

Here, $\mathrm{W}_{\mathrm{O}}=$ Initial weight, $\mathrm{Wt}=$ Wight after 25 revolution 
In vitro disintegration test: One tablet in each of the 6 tubes of the basket were taken and a disc to each tube were added and the temperature was maintained at $37 \pm 2^{0} \mathrm{C}$. The assemblies were raised and lowered between 30 cycles per minute. The time (in seconds) for complete disintegration of all the tablets in the apparatus were measured and recorded.

In vitro dissolution study: The dissolution rate was studied using USP type II tablet dissolution test apparatus with a paddle stirrer in $900 \mathrm{~mL}$ of $0.001 \mathrm{~N} \mathrm{HCl}$. A speed of $50 \mathrm{rpm}$ and a temperature of $37 \pm 0.5^{\circ} \mathrm{C}$ were used in each test. Aliquots were withdrawn at different time intervals, filtered and analyzed spectrophotometrically at $217 \mathrm{~nm}$ for Fexofenadine $\mathrm{HCl}$ against appropriate blank. To maintain a constant volume of dissolution medium, fresh medium equivalent to the volume of withdrawn sample was added immediately after withdrawal of the sample.

Characterization of release kinetics: In order to study the exact mechanism of drug release from the tablets, drug release data was analyzed according to zero order, Higuchi square root (Higuchi, 1963), Korsmeyer (Korsmeyer, 1983) kinetics.
MDT study: Mean dissolution time (MDT) was calculated from dissolution data using the following equation (Mockel. and Lippold., 1993):

$$
M D T=\left(\frac{n}{n+1}\right) k^{-\frac{1}{n}},
$$

Where, $\mathrm{n}=$ slope for Korsmeyer model

$\mathrm{k}=$ Korsmeyer constant

\section{Results and Discussion}

Physical parameter study: All the formulations showed uniform thickness. The average percentages of deviation of all tablet formulations were within the limit. In this study the percentage friability for all the formulations were below $1 \%$, indicating that the friability was within the prescribed limits. All the tablet formulations showed (Table 2) acceptable pharmacotechnical properties and complied with the compendial specifications for weight variation, hardness and friability, disintegration time.

Table 2. Physical properties of Fexofenadine HCl tablets.

\begin{tabular}{cccccc}
\hline Formulation & Weight $(\mathrm{mg})$ & Hardness $(\mathrm{kgf})$ & Thickness $(\mathrm{mm})$ & Friability (\%) & Disintegration time (Sec) \\
\hline F1 & $155.46 \pm 0.58$ & $2.54 \pm 0.03$ & $2.01 \pm 0.023$ & $0.4 \pm 0.010$ & 47 \\
F2 & $159.71 \pm 0.23$ & $2.63 \pm 0.09$ & $2.87 \pm 0.024$ & $0.3 \pm 0.032$ & 17 \\
F3 & $163.47 \pm 0.61$ & $2.7 \pm 0.18$ & $2.55 \pm 0.024$ & $0.5 \pm 0.010$ & 5 \\
F4 & $155.70 \pm 0.51$ & $2.26 \pm 0.11$ & $2.85 \pm 0.023$ & $0.2 \pm 0.012$ & 46 \\
F5 & $159.80 \pm 0.41$ & $2.70 \pm 0.02$ & $2.35 \pm 0.024$ & $0.5 \pm 0.067$ & 16 \\
F6 & $163.53 \pm 0.50$ & $2.58 \pm 0.14$ & $2.99 \pm 0.023$ & $0.4 \pm 0.023$ & 9 \\
F7 & $155.95 \pm 0.56$ & $2.45 \pm 0.30$ & $2.69 \pm 0.024$ & $0.3 \pm 0.050$ & 135 \\
F8 & $159.35 \pm 0.54$ & $3.23 \pm 0.10$ & $2.96 \pm 0.023$ & $0.4 \pm 0.025$ & 84.6 \\
F9 & $163.29 \pm 0.64$ & $3.48 \pm 0.14$ & $2.90 \pm 0.023$ & $0.2 \pm 0.056$ & 56 \\
F10 & $156.67 \pm 0.50$ & $2.47 \pm 0.14$ & $2.46 \pm 0.024$ & $0.4 \pm 0.014$ & 900 \\
F11 & $159.2 \pm 0.67$ & $3.38 \pm 0.22$ & $2.93 \pm 0.025$ & $0.4 \pm 0.010$ & 765 \\
F12 & $164.1 \pm 0.47$ & $3.63 \pm 0.16$ & $2.85 \pm 0.023$ & $0.4 \pm 0.075$ & 840 \\
F13 & $154.9 \pm 0.61$ & $3.11 \pm 0.14$ & $2.84 \pm 0.023$ & $0.09 \pm 0.016$ & 970.8 \\
F14 & $163.9 \pm 0.54$ & $3.47 \pm 0.19$ & $2.79 \pm 0.024$ & $0.4 \pm 0.025$ & 1590 \\
F15 & $155.50 \pm 0.47$ & $3.93 \pm 0.42$ & $2.69 \pm 0.024$ & $0.07 \pm 0.032$ & 1950 \\
\hline
\end{tabular}

It is clearly observed from comparing tables (Table 2) that incorporating of different concentration of disintegrant in the formulation definitely decreased the disintegration time to a noticeable extent. With the increase in concentrations of superdisintegrants, disintegration times were reduced significantly except Xanthan gum, which didn't act as disintegrant rather than it acts as binder (Figure 1). 


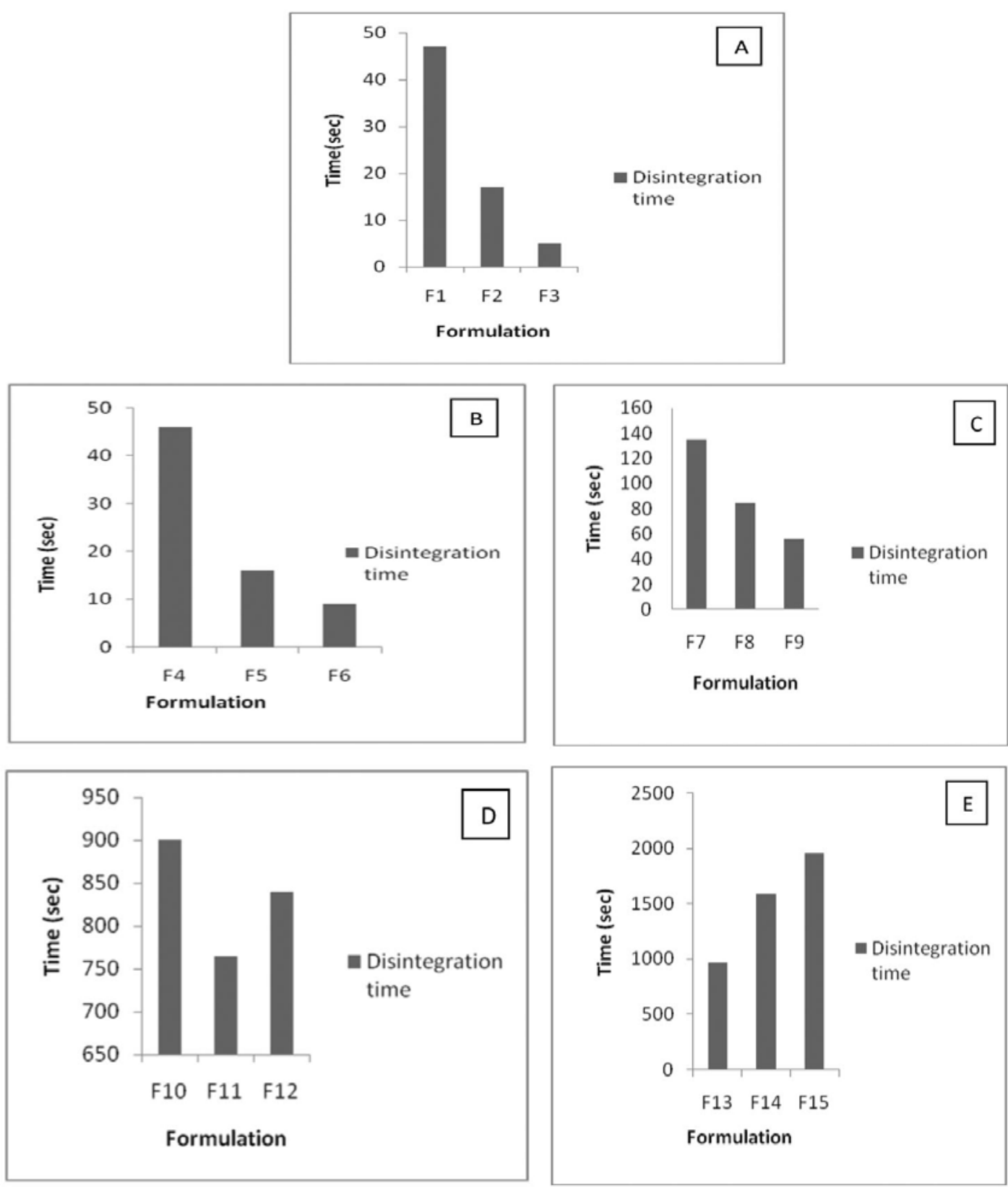

Figure 1. Bar diagram for disintegration time of various formulation. $\mathbf{A}=$ Formulations of Sodium starch glycolate, $\mathbf{B}=$ Formulations of Cross carmellose sodium, $\mathbf{C}=$ Formulations of crospovidone (kollidon CL), $\mathbf{D}=$ Formulations of ludiflash, $\quad \mathbf{E}=$ Formulations of Xanthan gum.

\section{Release profiles of Fexofenadine $\mathrm{HCl}$}

Dissolution study for tablets containing 3\% disintegrants: F-1 and F-4 formulations were released almost 84\% within 60 minutes, and other F-7, F-10, F-13 were released less than $60 \%$ at same time (Figure 2).

Dissolution study for tablets containing 6\% disintegrants: Formulation F-5 showed rapid drug dissolution, 95\% drug released within 60 minute and F-2 showed $86 \%$ drug released at the same time. On the other hand, formulation F-8 which contained Kollidon CL, showed $77 \%$ release in 60 minute (Figure 3 ).

Dissolution study for tablets containing 8\% disintegrants: The rapid drug dissolution observed from formulation F-3 and F-6 showed 99\% drug released within 
30 minutes and 45 minutes, respectively (Figure 4) than the market product which released $76 \%$ of drug at the end of 60 minutes. This rapid drug dissolution may be due to easy breakdown of particles and dissolution of drug into the medium. Thus the orally disintegrating tablets, apart from fulfilling all official and other specifications, exhibited faster release rates of Fexofenadine HCl.

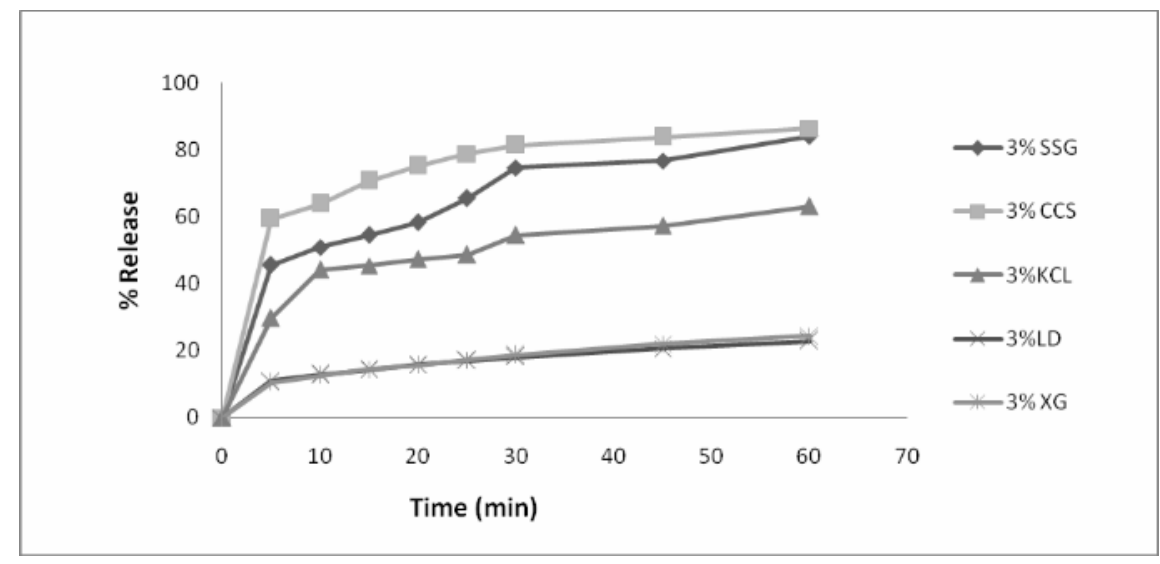

Figure 2. Zero order curve for F-1, F-4, F-7, F-10 and F-13

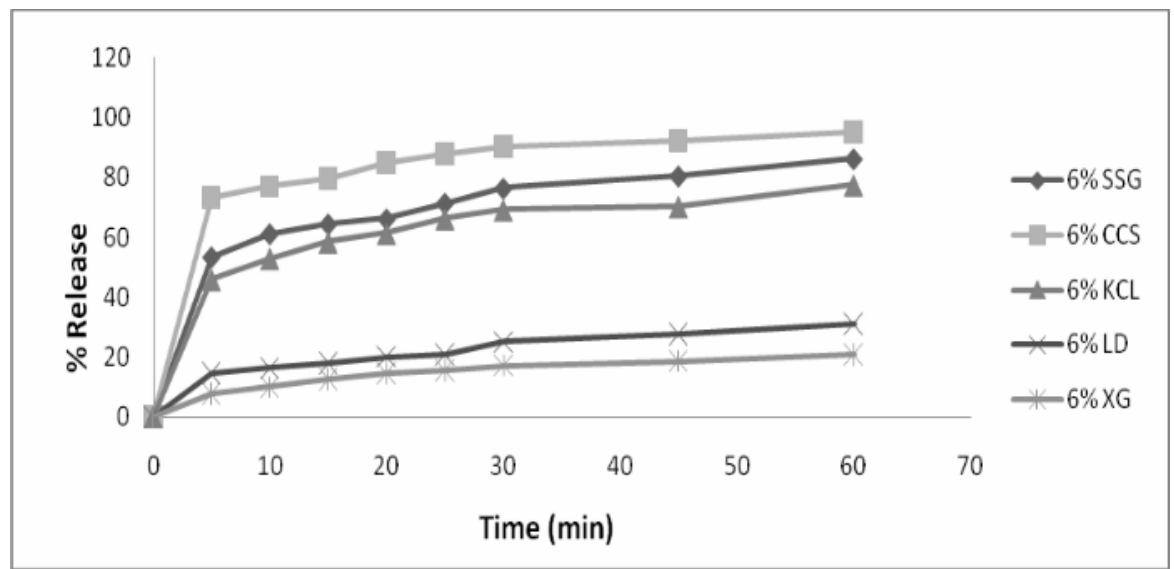

Figure 3. Zero order curve for F-2, F-5, F-8, F-11 and F-14.

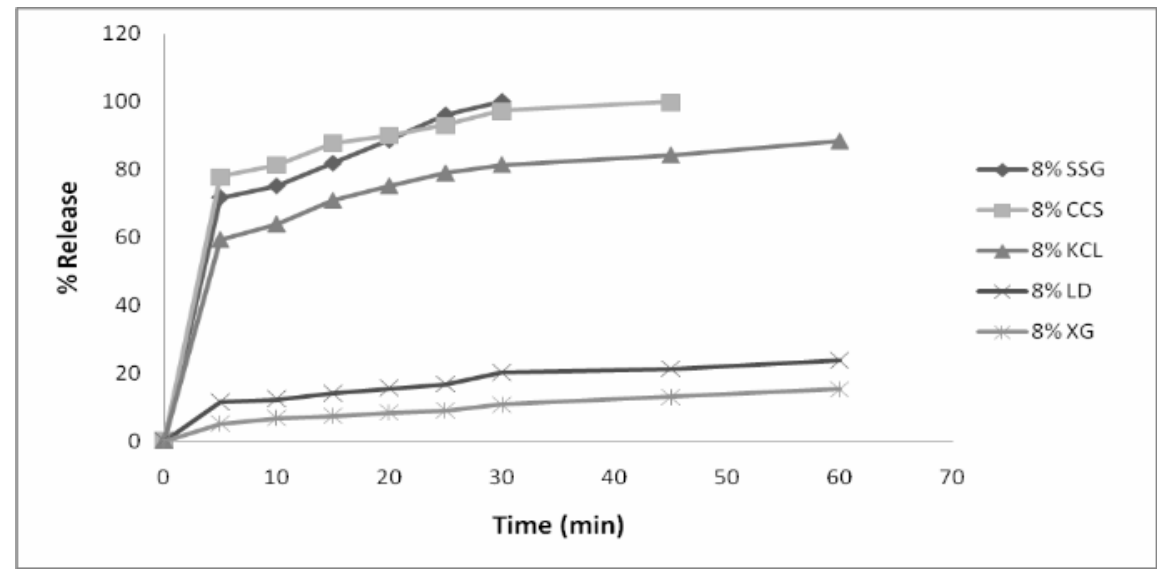

Figure 4. Zero order curve for F-3, F-6, F-9, F-12 and F-15. 
Best fitted model and their release mechanism: To investigate the effect of disintegrant agents on Fexofenadine $\mathrm{HCl}$ release fifteen formulations were made. From table $3, \mathrm{~F}-1$ best fits with Korsmeyer $\left(\mathrm{R}^{2}=0.949\right)$ then Higuchi $\left(\mathrm{R}^{2}=0.914\right)$. The values of release exponent obtain from Korsmeyer model is 0.261 which indicates that the release pattern of Fexofenadine $\mathrm{HCl}$ from $\mathrm{F}-1$ can be characterized by Fickian diffusion. In this case, the drug release is dominated by the diffusion mechanism. F-2 to F-9 also followed Korsmeyer model $\left(\mathrm{R}^{2}=0.982, \mathrm{R}^{2}=\right.$ 0.922, $\mathrm{R}^{2}=0.970, \mathrm{R}^{2}=0.968, \mathrm{R}^{2}=0.972, \mathrm{R}^{2}=0.926, \mathrm{R}^{2}=$ $0.984, R^{2}=0.980$ ), the release exponent values of these formulations were $0.192,0.193,0.163,0.121,0.113$, $0.270,0.208$ and 0.169 , these value indicates Fickian diffusion. Again F-10, F-11, F-12, F-13, F-14 and F-15 were found to best fit with Kosmeryer and also Higuchi model. The $\mathrm{R}^{2}$ values of Korsmeyer model for F-10, F-11, F-12, F-13, F-14 and F-15 were 0.990, 0.955, 0.947, $0.989,0.987$ and 0.973 respectively. The $R^{2}$ value of Higuchi for F-10, F-11, F-12, F-13, F-14 and F-15 were $0.950,0.960,0.955,0.975$ and 0.99 , respectively. The values of release exponent of Korsmeyer model for F-10, F-11, F-12, F-13, F-14 and F-15 were 0.297, 0.319, 0.318, $0.345,0.404$ and 0.430 , respectively, which denoted that Fickian diffusion phenomenon dominates. For each disintegrating agent the values of diffusion exponent (n) was reduced with the increase of disintegrant load which indicated the shifting of release mechanism except F-13 , F-14 and F-15 which contained Xanthan gum.

Table 3. Kinetic parameters of different formulation of Fexofenadine HCl tablet.

\begin{tabular}{cccccccc}
\hline Formulation & \multicolumn{2}{c}{ Zero order } & \multicolumn{2}{c}{ Higuchi } & \multicolumn{2}{c}{ Korsmeyer } & MDT (min) \\
\cline { 2 - 7 } & $\mathrm{k}_{0}$ & $\mathrm{R}^{2}$ & $\mathrm{k}_{\mathrm{H}}$ & $\mathrm{R}^{2}$ & $\mathrm{R}^{2}$ & $\mathrm{n}$ & 25.81 \\
F1 & 1.063 & 0.687 & 10.14 & 0.914 & 0.949 & 0.261 & 22.27 \\
F2 & 0.994 & 0.573 & 9.965 & 0.841 & 0.982 & 0.192 & 5.66 \\
F3 & 2.586 & 0.677 & 17.11 & 0.898 & 0.922 & 0.193 & 17.57 \\
F4 & 0.959 & 0.489 & 10.03 & 0.782 & 0.970 & 0.163 & 8.98 \\
F5 & 0.975 & 0.415 & 10.55 & 0.710 & 0.968 & 0.113 & 4.85 \\
F6 & 1.544 & 0.479 & 13.70 & 0.771 & 0.972 & 0.121 & 67.23 \\
F7 & 0.776 & 0.640 & 7.573 & 0.890 & 0.926 & 0.270 & 34.97 \\
F8 & 0.899 & 0.574 & 9.049 & 0.848 & 0.984 & 0.208 & 16.79 \\
F9 & 0.985 & 0.508 & 10.20 & 0.796 & 0.980 & 0.169 & 2152.19 \\
F10 & 0.295 & 0.745 & 2.764 & 0.95 & 0.990 & 0.297 & 640.83 \\
F11 & 0.416 & 0.787 & 3.805 & 0.960 & 0.955 & 0.319 & 1453.40 \\
F12 & 0.318 & 0.772 & 2.932 & 0.955 & 0.947 & 0.318 & 994.27 \\
F13 & 0.330 & 0.805 & 3.014 & 0.976 & 0.989 & 0.345 & 1067.43 \\
F14 & 0.294 & 0.794 & 2.705 & 0.975 & 0.987 & 0.404 & 1677.43 \\
F15 & 0.216 & 0.896 & 1.886 & 0.990 & 0.973 & 0.430 & \\
\hline
\end{tabular}

Mean dissolution time (MDT) study: From the table 3, it is clear that MDT values were changed due to the change of the amount of disintegrants in the tablets. In all these formulations, the values of MDT are larger for those formulations which contain smaller quantities of disintegrating agent. For example MDT values for F-1, F2 were 25.81 minutes and 22.27 minutes which contain $3 \%$ and $6 \%$ concentration of disintegrant, respectively but for F-3 was 5.66 minutes. So this reduction of the magnitude of MDT is most valuable observation which indicates that when the amount of sodium starch glycolate was increased, the drug release rate was increased gradually due to the disintegrating effect of the Sodium starch glycolate and the same picture was observed in case of formulations F-4 to F-9 where Cross carmellose sodium, Kollidon CL were used as disintegrants. In case of formulations F-13, F-14 and F-15 it was found that when the amount of xanthan gum were increased then the drug release rates were decreased, because xanthan gum didn't act as disintegrant in formulation at high concentration. 

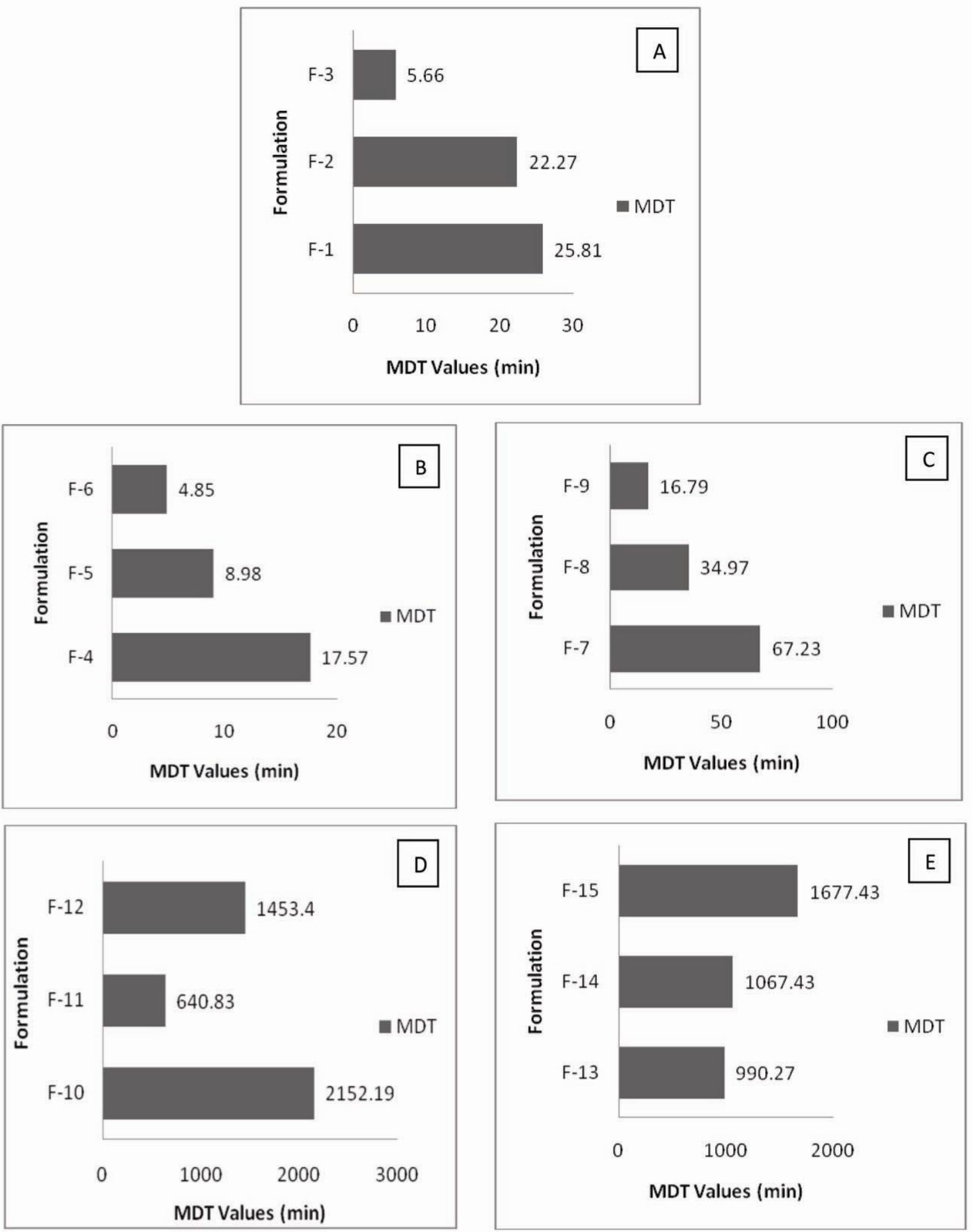

Figure 5. MDT values for various formulations. A=Formulations of sodium starch glycolate, $\mathbf{B}=$ Formulations of Cross carmellose sodium, $\mathbf{C}=$ Formulations of kollidon CL, $\mathbf{D}=$ =Formulations of ludiflash, $\mathbf{E}=$ Formulations of Xanthan gum 
Fourier transform infrared spectroscopic (FTIR) study: FTIR spectra were recorded to examine drugpolymer interactions. FTIR of pure Fexofenadine $\mathrm{HCl}$ is shown in figure 6. It shows $-\mathrm{NH}$ stretching at 3301.28 $\mathrm{cm}^{-1}$, C-O stretching at $1705 \mathrm{~cm}^{-1}, \mathrm{C}=\mathrm{O}$ stretching at
$1451.04 \mathrm{~cm}^{-1}$ and C-N stretching at $1278.68 \mathrm{~cm}^{-1}$. These peaks are identical for Fexofenadine $\mathrm{HCl}$. These peaks were found unchanged in F-3, F-6, F-9, F-11 and F-14 which are shown in figures $7,8,9,10,11$, respectively. This proves that there was no drug-polymer interaction.
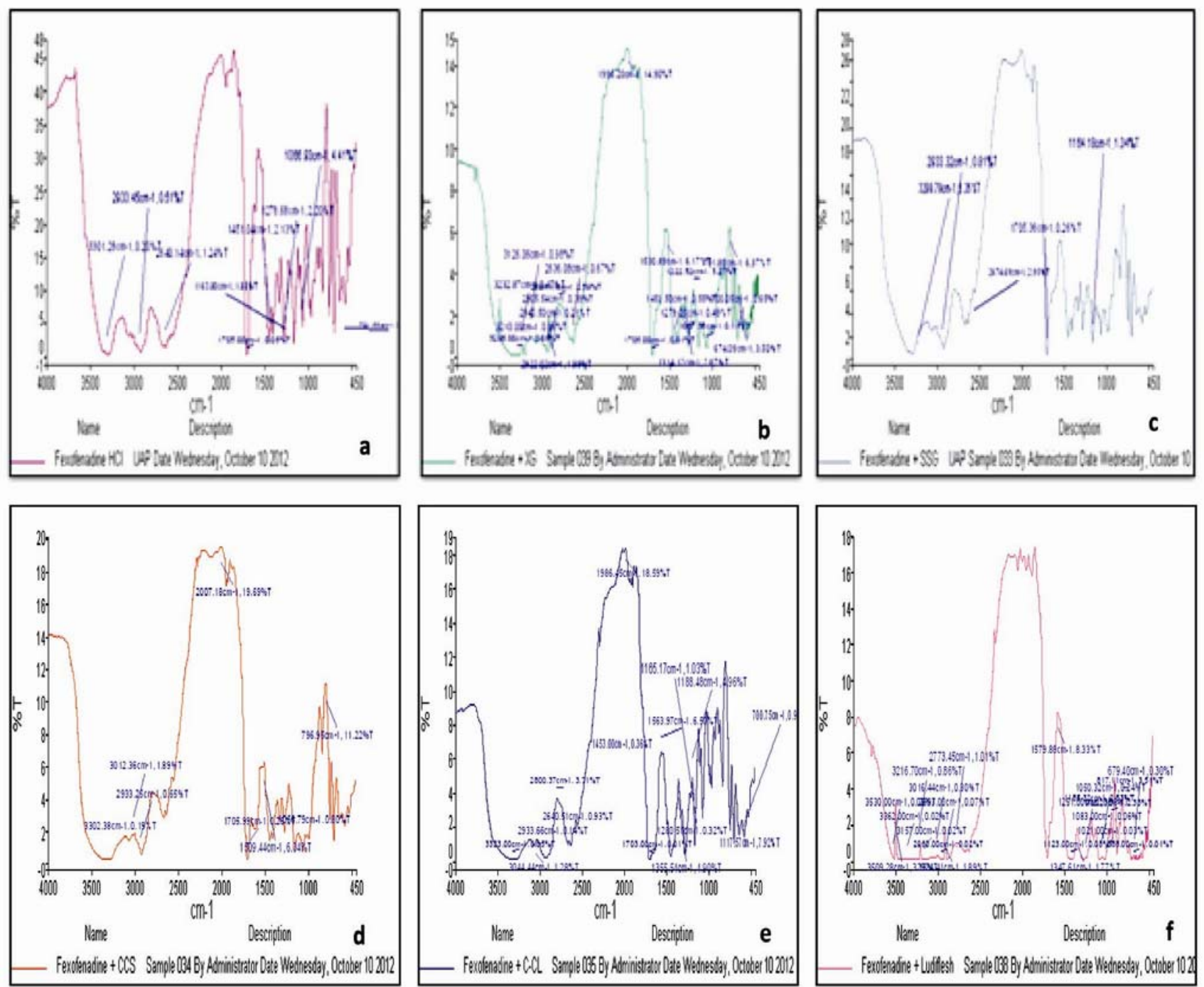

Figure 6. a, b, c, d, e and f represent IR spectra of pure FFN (Drug), FFN formulation with 8\% XG, FFN formulation with 8\% SSG, FFN formulation with 8\% CCS, FFN formulation with 8\% KCL and FFN formulation with 8\% Ludiflash, respectively.

\section{Conclusions}

In this study, tablets of Fexofenadine $\mathrm{HCl}$ were prepared by direct compression, where SSG, CCS, KCL, Xanthan Gum and Ludiflash were used as disintegrants. The release mechanism was explored and explained with Zero order, Higuchi and Korsmeyer model. Release profiles showed a tendency to diffusion mechanism.
Disintegration time of F-3, F-6 formulations were within 9 seconds because of the presence of $8 \%$ superdisintegrant of sodium starch glycolate and cross carmellose sodium, respectively, which resulted in improved rate and extent of dissolution. This was supported by the percent release of the formulations. Comparison of the release profile of the 15 formulations showed that formulation F-3 and F-6 was 
superior as compared to other formulations. On the other hand, Xanthan gum did not act as a superdisintegrant rather than it acted as a binder hence decreased the release of drug from respective formulations. So, it is suggested that the studied superdisintegrants (SSG, CCS) can be incorporated (in various concentrations) in the formulation by direct compression method to obtain desired result of Fexofenadine $\mathrm{HCl}$.

\section{References}

Aulton, M.E. 2002. Modified release per oral dosage forms, Pharmaceutics - The Science of Dosage form Design. $2^{\text {nd }}$ ed. New York: Churchill Livingstone.

Bhowmik, D., Chiranjib. B., Chandira, R.M. and Kumar, K.P. 2010. Emerging trends of disintegrants used in formulation of solid dosage from. Der Pharmacia lett. 2, 495-504.

Higuchi, T. 1963. Mechanism of sustained action medication, theoretical Analysis of rate of release of solid drug dispersed in solid matrics. J. Pharm. Sci. 52, 1145-1149.

Lachman, L., Lieberman, A.H. and Kanig, A.J. 1986. The theory and practice of Industrial Pharmacy. Lippincott Williams \& Wikins. $3^{\text {rd }}$ edi.

Lang, S., 1982. Effect of disintegrant incorporation on drug release. Manufacturing Chemistry 3, 31-32.

Lappin, G., Shishikura, Y., Jochemsen, R., Weaver, R.J., Gesson, C., Houston, B., Oosterhuis, B., Bjerrum, O.J., Rowland, M. and Garner. C. 2010. Pharmacokinetics of Fexofenadine: evaluation of a microdose and assessment of absolute oral bioavailability. Eur. J. Pharm. Sci. 40, 125-31.
Mockel, J.E. and Lippold, B.C. 1993. Zero order release from hydrocolloid matrices. Pharm. Res. 10, 1066-1070.

Peppas, N.A., 1985. Analysis of Fickian and non-Fickain drug release from polymers. Pharm. Analyt. Acta . 60, 110 -111. Philadelphia, USA, 1976: 293.

Parthiban, K.G., Nagarjun, K. and Kumar, N. 2011. Study of the impact of mode of incorporation of superdisintegrants in the dissolution of oral dosage forms. Int. J. Pharm. Res. Dev. 3, 70-76.

Polli, J. 2008. In vitro studies are sometimes better than conventional human pharmacokinetic In-Vivo studies in assessing bioequivalence of immediate-release solid oral dosage forms. AAPS. Pharma. Sci. Tech. 10, 289-299.

Raymond, C.R., Paul, J.S. and Paul, J.W. 2003. Handbook of Pharmaceutical Excipients. Pharmaceutical Press and American Pharmaceutical Association. $4^{\text {th }}$ ed. 108, 181, 184, 581, 691.

Shangraw, R., Mitrevej, A. and Shah, M. 1980. New era of tablet disintegrants. Pharm. Technol. 4, 49-57.

Sekulovic, D., Tufegdzic, N. and Birmanevic, M. 1986. The investigation of the influence of Explotab on the disintegration of tablets. Pharmazie 41, 53-154.

Zhao, N. and Augsburger, L.L. 2005. Functionality comparison of 3 classes of superdisintegrants in promoting aspirin tablet disintegration and dissolution. AAPS. Pharm. Sci. Tech. 6, E634-E640. 